\title{
DESIGN OF A NEARLY ZERO-ENERGY HOME WITH EXTREME COLLABORATION IN BIM
}

Alberto Nope Bernal

Universidad La Gran Colombia | Colombia | yuber.nope@ugc.edu.co

XXIV International Conference of the Iberoamerican Society

of Digital Graphics

Anna Gabriela Ramírez

Universidad La Gran Colombia | Colombia | anna.ramirez@ugc.edu.co

Rodrigo García Alvarado

Universidad del Bío Bío | Chile | rgarcía@ubb.cl

Eric Forcael Durán

Universidad del Bío Bío | Chile | eforcael@ubiobio.cl

\begin{abstract}
The global request of energy politics and actions against climate change, reiterate the importance of promoting the nearly zero-energy buildings (nZEB), taking into account environmental habitability and comfort; therefore, this type of buildings has to include a process, design, and construction, intelligent. Accordingly, the present research shows a methodology for the design of almost zero-energy housing, by using BIM under an environment of extreme collaboration; evaluating energy consumption and active solar generation. Thus, the proposed methodology allows optimizing the processes related to design time, level of geometric development, and the application and evaluation of sustainability strategies, to achieve nearly zero-energy housing within the city Concepción Chile.
\end{abstract}

Keywords: Nearly Zero-energy Buildings; Building Information Modeling; Extreme Collaboration; Sustainability

\section{INTRODUCCIÓN}

En noviembre de 2009, la Agencia Internacional de la Energía (AIE) presentó el estudio "World Energy Outlook" (WEO), donde se revisan las perspectivas energéticas hasta el año 2030. La AIE destaca que la tendencia actual tanto de suministro como de consumo de energía es insostenible, no sólo desde el punto de vista ambiental sino también desde el punto de vista económico y social (Ambiente \& Madrid, 2010). En la misma dirección, la tendencia global en políticas energéticas y las acciones frente al cambio climático, reiteran la importancia de lograr el objetivo de que las nuevas edificaciones sean de energía casi nula (Collado et al., 2019). Esto requiere de la optimización del rendimiento energético, a partir de un enfoque de diseño integrado y un trabajo en equipo interdisciplinario que optimice el uso de energía y la calidad del entorno interior del edificio a la vez que se satisfacen las necesidades de los ocupantes. (Trebilcock, 2009). En este sentido, el concepto de Building Information Modeling (BIM) es esencial para alcanzar estándares de sostenibilidad y eficiencia energética en la arquitectura, dado que, plantea que el proceso debe evolucionar de un modelo tradicional donde el proyecto transita de forma lineal (arquitecto - especialistas), hacia un proceso integrado donde todos los miembros del equipo de diseño trabajan en forma interdisciplinar y colaborativa desde las etapas iniciales. (Forcael et al., 2020; Kwok et al., 2015).

Por su parte, las altas exigencias técnico constructivas de las edificaciones cero energía, requieren establecer un método colaborativo que permita abordar la sostenibilidad social, ambiental y económica en etapas tempranas de diseño, con impacto en todas las etapas del ciclo de vida de la edificación (Parasonis et al., 2012). Es así como, el diseño de una vivienda cero energía en un clima frío húmedo, - como el que se encuentra en lugares como el centro sur de Chile-, supone un reto para el cumplimiento de normativas locales de viviendas eficientes energéticamente.

El objetivo de la presente investigación fue evaluar el grado de sostenibilidad ambiental y balance energético de una vivienda cero energía, a través de una metodología basada en la interacción temprana entre especialistas de arquitectura, sostenibilidad, estructura y especialidades MEP (Mechanical, electrical and plumbing) en un ambiente de colaboración extrema (Figura 1), reconociendo indicadores, estrategias bioclimáticas y de eficiencia energética, para equilibrar demandas por climatización y aporte solar activo, respetando condiciones de confort ambiental y habitabilidad.

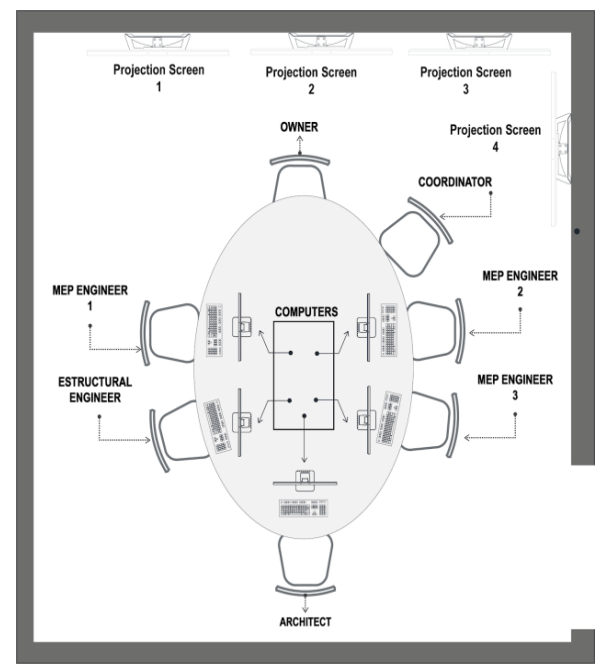

Figura 1. Ambiente de Colaboración Extrema. Laboratorio de Diseño Integrado de Proyectos Universidad del Bio Bio 


\section{EDIFICIOS DE ENERGÍA CASI CERO}

La comunidad europea es pionera en el desarrollo de estándares de edificaciones bajas en carbono, estableciendo para el año 2020 la ambiciosa meta de lograr valores demanda energética para edificios residenciales entre $20 \mathrm{kWh} / \mathrm{m} 2 / \mathrm{Año}$ a 180 kWh/m2/Año.(Piderit et al., 2019) De acuerdo a las regulaciones Nacionales, cada país establece los valores objetivo de acuerdo a las condiciones climáticas particulares, pese al gran esfuerzo en la implementación, el concepto nZEB, por sus siglas en inglés de "nearly zeroenergy Building" se ha venido difundiendo de manera lenta y algunos países no han establecido la definición del concepto y no se ha logrado trabajar una regulación que permita la máxima difusión y práctica en los edificios construidos. En términos generales puede considerarse que un" edificio de energía casi cero" es un edificio que tiene un muy alto rendimiento energético; el casi cero o una cantidad muy baja de energía requerida debe cubrirse en gran medida con energía de fuentes renovables (Kurnitski et al., 2011)

Para el caso de la región latinoamericana el alcance de los objetivos del concepto nZEB, se vislumbra alejados puesto que para muchos países no existe regulación que permita acercarse a la arquitectura baja en emisiones. Algunos países han trabajado en normativas que permiten el ahorro de energía en las edificaciones tal es el caso de Chile, Argentina, Brasil, México; sin embargo, aún existe un vacío normativo. Por lo que la construcción sustentable hace parte de esfuerzos puntuales, voluntarios y se ve reflejada principalmente por la inclusión de criterios de sustentabilidad establecidos en estándares y certificaciones provenientes de otras latitudes.

Existe poco acuerdo sobre la definición de nZEB, el término se ha usado comercialmente sin un entendimiento claro y los países han promulgado políticas y metas nacionales basadas en el concepto sin una clara definición; sin embargo, existe cierta convergencia basada en el análisis científico, que tiene que ver con los criterios de energía primaria y emisiones de carbono, considerados en el balance entre la energía necesaria para la operación de los edificios, la energía embebida en los materiales de construcción y las instalaciones técnicas. Las diversas definiciones sugieren diferentes estrategias, influencia en las elecciones de aislamiento térmico, sistemas de aire acondicionado, y dimensionamiento de sistemas fotovoltaicos o de cogeneración (Joanna et al., 2010) (Asaee et al., 2019)

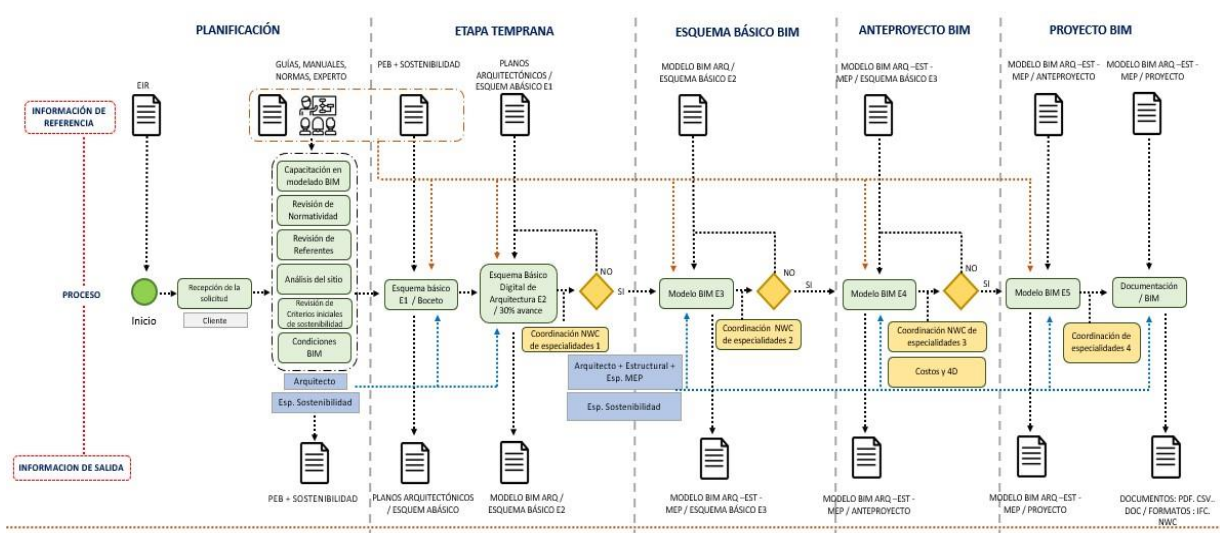

Figura 2. Mapa de proceso para el diseño de vivienda casi cero energía.
La definición del nZEB y ZEB no es muy clara y puede variar de acuerdo con la localización y país donde se desarrolle, por sus condiciones climáticas, la industria de la construcción y la realidad socioeconómica hace que se pueda adaptar a las normativas de cada país. En general se sabe que estos conceptos requieren de una alta eficiencia energética y el uso de energías renovables, dependiendo de la definición es obligatorio que sea en sitio o en otras es indiferente el lugar en donde se produzca la energía. En lo que parece haber un común acuerdo es que estándares como Passivhaus o Cassaclima, Klimahaus, son edificaciones que se consideran de consumo energético casi nulo, en la forma básica de cumplimiento de los mismos. Una definición es la de medición del balance que se puede hacer por la energía, el co2, el costo de la energía u otros parámetros definidos por la política energética nacional, esto puede ser para diferentes periodos de tiempo, como su ciclo de vida total, su periodo de uso o su consumo anual. (Marszal et al., 2011)

\section{METODOLOGÍA}

Para cumplir con el objetivo propuesto, se formuló una metodología que consideró la elaboración de un mapa de proceso (Figura 1) en el cual se estructuró la secuencia de trabajo, partiendo desde la etapa de planeación hasta diseño. Una vez estructurado el mapa, y mediante el Laboratorio de Diseño integrado de Proyectos, se inició el diseño de una vivienda casi cero-energía, ubicada en la localidad de Coliumo, ubicada en el centro-sur de Chile. Este ejercicio de diseño se logró en seis semanas, con tres sesiones por cada una para un total de 9 horas semanales, con el equipo de trabajo relacionado en la Figura 2 y Tabla 1.

\section{MAPEO DE PROCESO}

El mapeo de proceso es un diagrama de valor que representa, a manera de inventario gráfico, los procesos de una organización en forma interrelacionada y que, para este caso, permitió identificar, etapas de ciclo de vida (planeación, diseño básico, anteproyecto y proyecto), reconociendo procesos, interacciones, documentación de referencia y salida. Lo anterior para establecer aspectos y actividades que permitieran desarrollar el ejercicio de diseño de forma ordenada y estructurada. La Figura 2, muestra el mapeo realizado previo al inicio del diseño y modelado BIM de una vivienda casi cero-energía. 
Tabla 1. Roles y responsabilidades asignadas al equipo de trabajo.

\begin{tabular}{|c|c|c|c|c|}
\hline ESPECIALIDAD & PERFIL & ENTREGABLES & HORAS & SESIONES \\
\hline $\begin{array}{l}\text { Cliente / } \\
\text { Mandante }\end{array}$ & & $\begin{array}{l}\text { EIR (Employer } \\
\text { Information } \\
\text { Requirements) }\end{array}$ & 3 & 1 \\
\hline $\begin{array}{l}\text { Coordinador } \\
\text { de proyecto }\end{array}$ & Arquitecto & $\begin{array}{c}\text { Mapa de proceso } \\
\text { BEP+SOSTENIBILIDAD }\end{array}$ & & \\
\hline $\begin{array}{l}\text { Coordinador } \\
\text { Laboratorio }\end{array}$ & $\begin{array}{c}\text { Estudiante } \\
\text { de Ingeniería } \\
\text { Civil y } \\
\text { Ambiental } \\
\end{array}$ & $\begin{array}{c}\text { Configuración Ambiente } \\
\text { de Colaboración } \\
\text { Extrema y Diseño } \\
\text { Colaborativo. }\end{array}$ & 18 & 6 \\
\hline & ctudiante. & -Modelo Revit / MEP & & \\
\hline Sanitario & $\begin{array}{c}\text { Escuela de } \\
\text { Ingeniería en } \\
\text { Construcción }\end{array}$ & $\begin{array}{c}\text {-Cantidades de } \\
\text { materiales }\end{array}$ & 18 & 6 \\
\hline \multirow{3}{*}{ Arquitectura } & \multirow{3}{*}{$\begin{array}{l}\text { Estudiante: } \\
\text { Escuela de } \\
\text { Arquitectura }\end{array}$} & $\begin{array}{l}\text { Esquema básico digital } \\
\text { de diseño }\end{array}$ & \multirow{3}{*}{18} & \multirow{3}{*}{6} \\
\hline & & Modelo Revit / ARQ & & \\
\hline & & $\begin{array}{c}\text { Coordinación de } \\
\text { especialidades y } \\
\text { documentación de } \\
\text { proyecto. }\end{array}$ & & \\
\hline Sostenibilidad & $\begin{array}{c}\text { Estudiante } \\
\text { de Maestría }\end{array}$ & $\begin{array}{c}\text { Informe de confort, } \\
\text { consumo y generación. }\end{array}$ & 18 & 6 \\
\hline Eléctrico & $\begin{array}{l}\text { Estudiante: } \\
\text { Escuela de } \\
\text { Ingeniería } \\
\text { Civil y } \\
\text { Ambiental }\end{array}$ & Modelo Revit / MEP & 18 & 6 \\
\hline Estructural & & Modelo Revit /EST & 18 & 6 \\
\hline Mecánico & & Modelo Revit / MEP & 18 & 6 \\
\hline
\end{tabular}

PLANIFICACIÓN: esta etapa indica y define en primera instancia el EIR (Employer Information Requirements), el cual forma parte de los documentos de requisitos del cliente, en donde se establecen las condiciones con respecto al uso de la metodología BIM del proyecto, y que, para este caso, establece como principal objetivo, lograr una vivienda casi cero-energía. Una vez recibida la solicitud por el coordinador del proyecto, se identificaron los documentos y procesos previos a la elaboración del esquema básico E1 / Boceto. En esta etapa el Coordinador de Proyecto, formuló una etapa de capacitación en modelado BIM para el equipo de trabajo, compuesto por estudiantes de Arquitectura, Ingeniería Civil e Ingeniería en Construcción, (Figura 2). Asimismo, se hizo la revisión de normatividad, referentes, características del sitio, criterios de sostenibilidad y condiciones BIM (niveles de desarrollo, interoperabilidad, dimensiones y documentación). Lo anterior para consolidar un PEB (Plan de Ejecución BIM) + SOSTENIBILIDAD, que orientó el modelado de proyecto.

ETAPA TEMPRANA: a partir del análisis del lugar (clima, topografía, vialidad) y las necesidades espaciales y habitacionales del cliente, se desarrolló un programa de espacios y áreas que junto con la información consignada en el PEB + SOSTENIBILIDAD, permitió desarrollar los Esquema básico E1 / Boceto (Figura 4) considerando indicadores y recomendaciones de diseño sostenible como la orientación, factor de forma, inclinación de cubiertas, proporción de vidriado, ubicación de recintos y materialidad; para luego pasar al Esquema Básico Digital de Arquitectura E2 y su respectiva etapa de coordinación en Revit para revisar y comprobar interferencia e inconsistencias el mismo modelo de arquitectura, finalizando con la revisión general por parte del cliente/mandante, coordinador de proyecto, arquitectura y sostenibilidad.

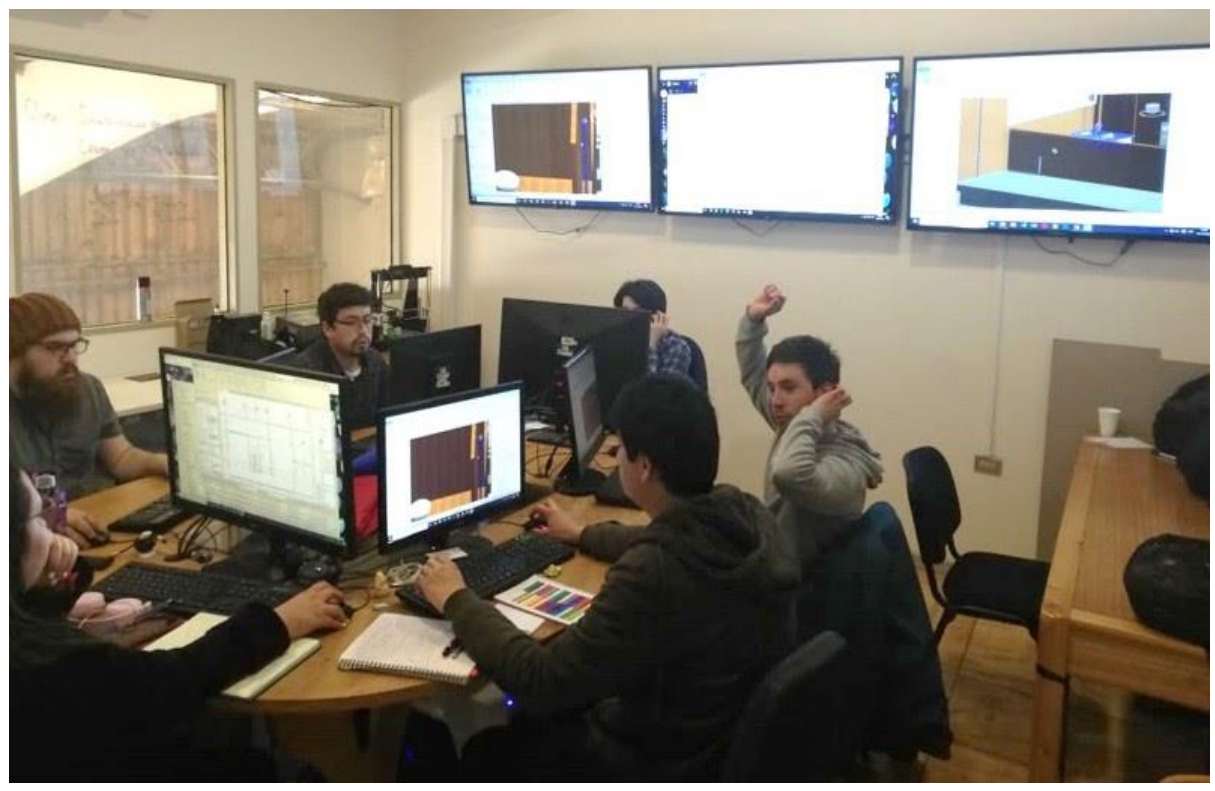

Figura 3. Equipo de trabajo en Ambiente de Colaboración Extrema. Laboratorio de diseño integrado de proyectos de la Universidad del Bio Bio 

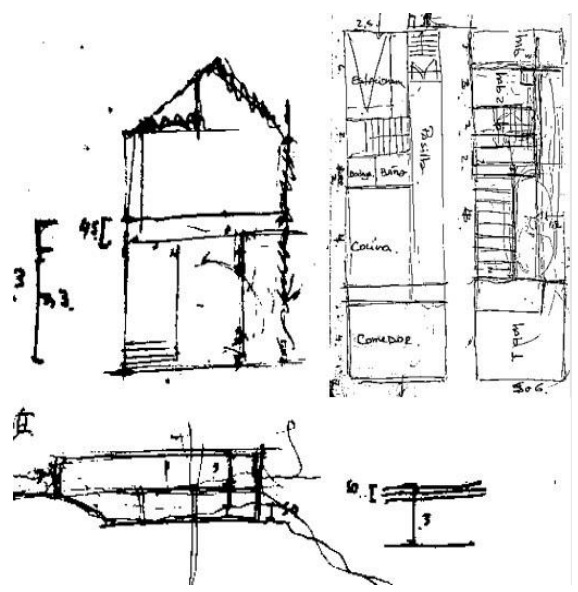

Figura 4. Esquema Básico E1 / Bocetos. Vivienda casi cero energía.

ESQUEMA BÁSICO BIM: en esta etapa se configuró la primera aproximación de diseño asistido por computadora, que relaciona arquitectura y sostenibilidad (Figura 5). Para cumplir con el objetivo de diseñar una vivienda ceroenergía, se realizaron simulaciones preliminares de consumo energético y potencial solar activo, empleando plataformas web como Insight 360. Esto permitió ajustar condiciones de forma, ubicación de espacios y parámetros como protección solar por fachada, materialidad de muros, cubierta y tipo de ventanas. Asimismo, se desarrollaron las primeras familias (muros, cubierta, losas, puertas y ventanas) con un LOD (Level of Development) 200 y un LOI (Level of Information) de 100; y se configuraron las habitaciones y espacios analíticos, tomando en cuenta condiciones de uso y operación (Figura 6), aspecto importante para la exportación de modelos BIM a programas especializados de simulación energética. Se define utilizar un sistema constructivo de placas de madera laminada CLT (cross-laminated timber), debido a su baja huella ambiental y buen desempeño estructural, térmico, acústico e ignifugo (Gong et al., 2019; Laguarda Mallo \& Espinoza, 2014; Pei et al., 2016).

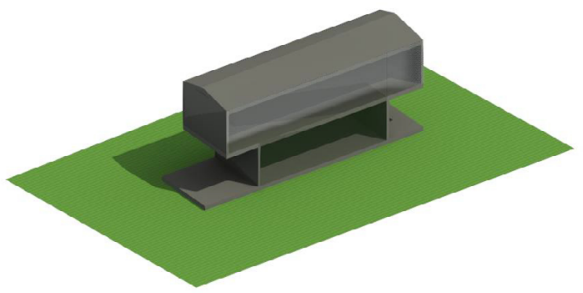

Figura 5. Esquema Básico Digital de Arquitectura E2. Vivienda cero casi cero-energía.

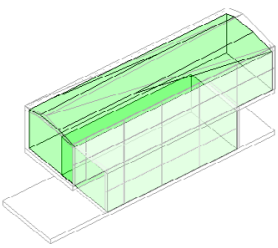

Figura 6. Zonas analíticas configuradas en Revit.

En esta etapa y considerando un nivel de avance del modelo arquitectónico, cercano al 30\%, se iniciaron los modelos de estructura y MEP (Figura 7), el que era analizado en tiempo real, mediante el trabajo de colaboración extrema y utilización de modelación en línea, en el cual los especialistas trabajaban en un modelo central, lo que permitió solucionar rápidamente problemas de interferencias entre instalaciones MEP, así como prever cruces de ductos y tuberías por muros y losas.

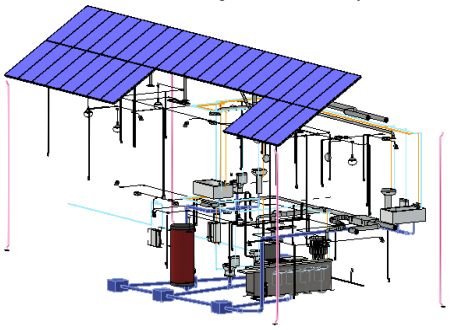

Figura 7. Modelo de instalaciones MEP

ANTEPROYECTO Y PROYECTO BIM: con un avance del $60 \%$ de los modelos, se ajustaron los niveles de LOD y LOI a 300 , esto para elementos de envolvente y espacios analíticos (Figura 8), los cuales mediante formato gbXML, se exportaron a Design Builder. De forma similar, para analizar el aporte solar activo, se exportó el modelo BIM/Revit a Autocad a través del formato DWG, para luego pasarlo a 3D Max y exportarlo a PVSol Premium como objeto (Figura 8), Lo anterior permitió obtener, resultados de confort ambiental (temperatura, humedad y calidad del aire), consumo energético y generación solar activo, a través de paneles solares fotovoltaicos consignados en el Gráfico 1 y las Tablas 4 y 5 .

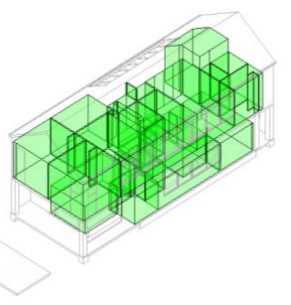

Figura 8. Modelos Design Builder y PVsol.

Las simulaciones de confort ambiental y consumo energético, se realizaron mediante simulaciones dinámicas utilizando el software Design Builder (el cual emplea como motor de cálculo Energy Plus) (Figura 8), utilizando los parámetros descritos en la Tabla 2; asimismo, para 
establecer el aporte solar activo mediante paneles solares fotovoltaicos, se realizaron simulaciones en PVSol Premium (Figura 8), incluyendo parámetros como, ubicación, tipo y eficiencia de paneles solares, tipo de conexión y superficie de cubierta y fachada (Tabla 3 ).
2018), para identificar indicadores y estrategias proyectuales de sostenibilidad a ser implementadas y validadas en la etapa inicial de diseño y anteproyecto respectivamente (Tabla 3 )

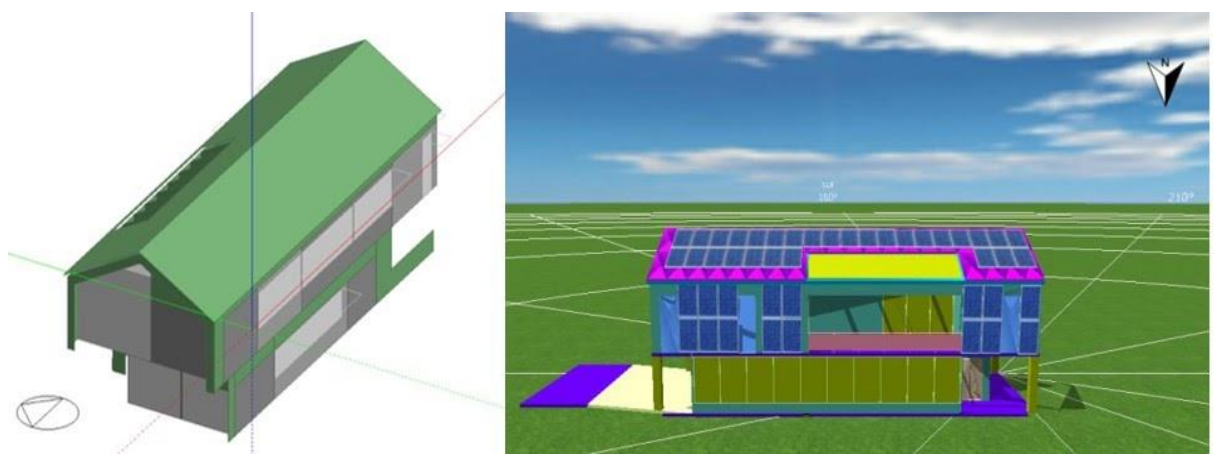

Figura 9. Modelos Design Builder y PVsol.

Por otro lado, todas las etapas de diseño consideraron instancias de Coordinación NWC de especialidades, las cuales permitían corroborar, verificar y solucionar interferencias, y hacer ajustes al diseño, del trabajo realizado en la experiencia de colaboración extrema para BIM. La Tabla 2, muestra las interferencias encontradas en cada una de las etapas y su estado de revisión y aprobación.

Tabla 2. Interferencias identificadas en cada instancia de coordinación.

\begin{tabular}{|l|c|c|}
\hline \multicolumn{1}{|c|}{ Etapa } & $\begin{array}{c}\text { Número de } \\
\text { interferencias }\end{array}$ & Informadas a \\
\hline $\begin{array}{l}\text { Etapa temprana / } \\
\text { Coordinación 1 }\end{array}$ & 65 & Arquitectura \\
\hline $\begin{array}{l}\text { Esquema Básico } \\
\text { BIM / } \\
\text { Coordinación 2 }\end{array}$ & 1969 & $\begin{array}{c}\text { Arquitectura y } \\
\text { Estructura }\end{array}$ \\
\hline $\begin{array}{l}\text { Anteproyecto BIM } \\
\text { / Coordinación 3 }\end{array}$ & 756 & $\begin{array}{c}\text { Estructura y } \\
\text { MEP }\end{array}$ \\
\hline $\begin{array}{l}\text { Proyecto BIM / } \\
\text { Coordinación 4 }\end{array}$ & 300 & MEP \\
\hline
\end{tabular}

\section{INDICADORES Y ESTRATEGIAS DE SOSTENIBILIDAD}

Se consultaron guías, manuales y estándares de sostenibilidad y eficiencia energética, como Términos de Referencia Estandarizados TDRe (CITECTUBB, 2015), Guía de diseño para la eficiencia energética en la vivienda social (Bustamante et al., 2009) y los Estándares de Construcción Sustentable para Viviendas en Chile (Minvu,
Tabla 3. Indicadores y estrategias de sostenibilidad implementadas

\begin{tabular}{|c|c|c|c|}
\hline Indicador & Valor & $\begin{array}{l}\text { Estrategia } \\
\text { pasiva }\end{array}$ & Estrategia activa \\
\hline $\begin{array}{l}\text { Iluminación n } \\
\text { / lux/m2 }\end{array}$ & 300 luxes & $\begin{array}{c}\text { Orientación } \\
- \\
\text { porcentaje } \\
\text { de vano- } \\
\text { volumetría }\end{array}$ & $\begin{array}{c}\text { Tipo y } \\
\text { distribución de } \\
\text { lámparas }\end{array}$ \\
\hline $\begin{array}{l}\text { Temperatura } \\
I^{\circ} \mathrm{C}\end{array}$ & $20-26^{\circ} \mathrm{C}$ & $\begin{array}{c}\text { Orientación } \\
\text { - Factor de } \\
\text { Forma - } \\
\text { Materialidad } \\
- \\
\text { Proporción } \\
\text { de vidriado }\end{array}$ & Bomba de calor \\
\hline $\begin{array}{l}\text { Calidad del } \\
\text { aire }\end{array}$ & $\begin{array}{c}2.3 \mathrm{l} / \mathrm{s} \\
\text { persona }\end{array}$ & - & $\begin{array}{l}\text { Intercambiador } \\
\text { de aire }\end{array}$ \\
\hline $\begin{array}{l}\text { Humedad } \\
\text { Relativa \% }\end{array}$ & $\begin{array}{c}\operatorname{Min} 30 \% \\
\text { max60\% } \\
\text { (ASHRAE)- }\end{array}$ & - & Deshumidificador \\
\hline $\begin{array}{l}\text { Demanda / } \\
\mathrm{KWh} / \mathrm{m} 2 \text { (año) }\end{array}$ & 20 & Anteriores & $\begin{array}{l}\text { Paneles solares } \\
\text { fotovoltaicos } \\
\text { integrado en } \\
\text { cubierta y } \\
\text { fachada norte. }\end{array}$ \\
\hline
\end{tabular}

Las decisiones de diseño se tomaron de acuerdo a los objetivos y estándares de construcción consultados estableciendo objetivos en la etapa temprana de diseño. Con lo anterior se definieron las características de envolvente, equipos, forma y disposición solar, a utilizar en las simulaciones de confort, demanda energética y aporte solar activo, como lo indican las tablas 4 y 5 . 
Tabla 4. Parámetros de simulación utilizados en Design Builder.

\begin{tabular}{|l|l|}
\hline \multicolumn{2}{|c|}{ Caracterización de proyecto } \\
\hline \multicolumn{2}{|c|}{ MATERIALIDAD } \\
\hline MUROS & CLT (valor U: 0.97 W/m2-K) \\
\hline CUBIERTA & CUBIERTA VERDE - TEJA ASFÁLTICA \\
\hline ESCALERA & CLT (valor U: 0.97 W/m2-K) \\
\hline Exteriores nivel 2 & CLT (valor U: 0.97 W/m2-K) \\
\hline Exteriores nivel 1 & Tablón Flexible \\
\hline CIELOS & Baldosas de piedra(negro) \\
\hline MOBILIARIO & Yeso Cartón \\
\hline PUERTAS & Madera \\
\hline VENTANAS & Madera - Aluminio - Vidrio \\
\hline BARANDILLA & Doble Vidriado hermético \\
\hline RAMPA & Aluminio - Vidrio \\
\hline & Piedra - Hormigón \\
\hline NIVEL 1 & 84,46 \\
\hline NIVEL 2 & 136,82 \\
\hline SUP. TOTAL & 221,28 \\
\hline ÁREA HABITABLE & 93 m2 \\
\hline CARGA DE OCUPACIÓN 4 PERSONAS= 0,043 pers/m2 \\
\hline
\end{tabular}

Tabla 5. Parámetros de simulación PVSol Premium.

\begin{tabular}{|c|l|}
\hline Tipo de panel & $\begin{array}{l}\text { Módulo activo } 200 \mathrm{wp} \\
\text { - si polycrystaline } 200 \mathrm{w} \\
\text { erecto }\end{array}$ \\
\hline Cantidad en cubierta norte & 29 \\
\hline Cantidad en fachada norte & 16 \\
\hline Tipo de sistema & Autónomo \\
\hline
\end{tabular}

\section{RESULTADOS}

Finalmente, con los modelos y simulaciones terminadas, se realizó toda la documentación. Para los datos de confort se obtuvo una temperatura operativa anual de $20,87^{\circ} \mathrm{C}$, registrando en invierno $19,7^{\circ} \mathrm{C}$ y en verano $26^{\circ} \mathrm{C}$. La humedad relativa se encuentra entre $47.2 \%$ y $55.8 \%$, como lo indica la gráfica 1; El balance energético de la vivienda, de acuerdo a la tabla 6 . Indica una pérdida total de energía anual de 7,648.12 KWh, debido a la orientación sentido norte de la vivienda las ganancias solares ascienden a $17,218.51 \mathrm{KWh}$, por lo que la demanda de energía para calefacción es de 4,960,47 Kwh, la de refrigeración es $459,83 \mathrm{Kwh}$, con un total de $58.28 \mathrm{kwh} / \mathrm{m} 2$ Año.

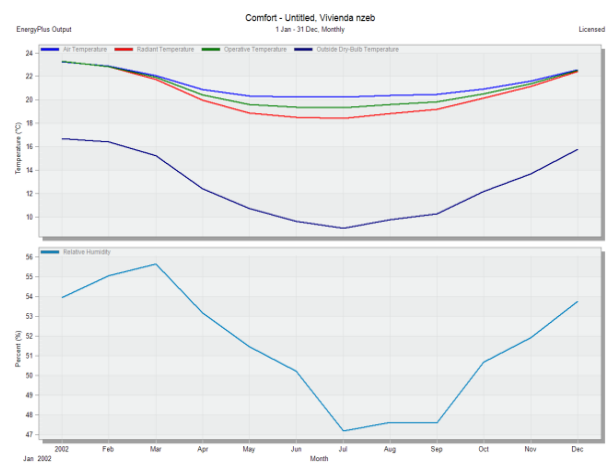

Gráfico 1. Resultados de Confort ambiental.

Tabla 6. Balance Energético Resultado

\begin{tabular}{|l|l|l|}
\hline \multicolumn{3}{|c|}{ BALANCE ENERGÉtICO MODELO DESIGN BUILDER } \\
\hline Ventilación + Infiltración & $\mathrm{ac} / \mathrm{h}$ & 0,93 \\
\hline Infiltraciones & $\mathrm{kWh}$ & $-7648,12$ \\
\hline Iluminación & $\mathrm{kWh}$ & 3556,49 \\
\hline Equipos & $\mathrm{kWh}$ & 7301,02 \\
\hline Ocupación & $\mathrm{kWh}$ & 8808,29 \\
\hline Ganancias Solares & $\mathrm{kWh}$ & 17218,51 \\
\hline $\begin{array}{l}\text { Temperatura del Aire } \\
\text { Anual }\end{array}$ & ${ }^{\circ} \mathrm{C}$ & 21,31 \\
\hline $\begin{array}{l}\text { Temperatura Radiante } \\
\text { Anual }\end{array}$ & ${ }^{\circ} \mathrm{C}$ & 20,43 \\
\hline $\begin{array}{l}\text { Temperatura operativa } \\
\text { anual }\end{array}$ & ${ }^{\circ} \mathrm{C}$ & $\mathbf{2 0 , 8 7}$ \\
\hline Temperatura exterior & ${ }^{\circ} \mathrm{C}$ & 12,64 \\
\hline Demanda de Calefacción & $\mathrm{kWh}$ & 4960,47 \\
\hline Demanda de Refrigeración & $\mathrm{kWh}$ & $\mathbf{4 5 9 , 8 3}$ \\
\hline Demanda Climatización & $\begin{array}{l}\mathrm{kWh} / \mathrm{m} \mathbf{2} \\
\mathrm{Anno}\end{array}$ & $\mathbf{5 8 , 2 8}$ \\
\hline
\end{tabular}

De acuerdo con los datos obtenidos en la estimación de producción de energía fotovoltaica (Tabla 7), para el área de $93 \mathrm{~m} 2$ habitables, los $44.34 \mathrm{KWh} / \mathrm{m} 2$ logran suplir el $76.1 \%$ de la demanda anual para climatización de la vivienda, los $13.94 \mathrm{KWh} / \mathrm{m} 2$, ubican a la vivienda en los rangos de demanda estimada para edificaciones de consumo energético cercano a cero. Aunque se debe reconocer que el desfase temporal (diario/estacional) de la energía generada vs consumida debe suplirse con baterías o a través de intercambio por la red, con subsecuentes pérdidas. 
Tabla 7. Generación Solar activa

\begin{tabular}{|l|l|l|}
\hline Energia FV utilizable & 4.124 & KWh (Año) \\
\hline Superficie de cubierta & 65 & $\mathrm{M} 2$ \\
\hline $\begin{array}{l}\text { Superficie habitable } \\
\begin{array}{l}\text { Relación FV / m2 } \\
\text { Habitable }\end{array}\end{array}$ & 93 & $\mathrm{M} 2$ \\
\hline
\end{tabular}

\section{DISCUSIÓN}

Una estrategia de diseño, basada en mapas de proceso, revisión de indicadores, criterios de sostenibilidad y uso de metodologías de trabajo colaborativo como BIM, permite mejorar los tiempos de diseño, comunicación y coordinación entre especialista, logrando edificaciones energéticamente eficientes y confortables, lo que puede ser replicado como estrategia en oficinas de diseño y laboratorios académicos de sostenibilidad.

Esta investigación ha permitido revisar cambios de paradigmas respecto a la formación del arquitecto, constructor y los especialistas que intervienen en el diseño, construcción y operación de proyectos más sostenibles. El desarrollo de una metodología de trabajo multidisciplinar permitió tomar decisiones acertadas en etapas tempranas de diseño, mediante objetivos claros de sostenibilidad que permitieron el máximo ahorro energético integrando la energía solar fotovoltaica como fuente primaria de energía para el confort térmico interior.

El trabajo desarrollado en un modelo 3D central, en línea, permitió optimizar el tiempo de modelado y la obtención de resultados en el tiempo estimado para las sesiones colaborativas, la integración de criterios de sostenibilidad permitió evidenciar que si bien los retos que propone la incorporación de la metodología BIM en el diseño de edificaciones sustentables requieren una formación previa ; la optimización de procesos que permiten los ambientes de colaboración extrema representan una oportunidad para involucrar la gestión en las etapas previas y post sesiones, representando cada una, un pilar en la gestión y ejecución del proceso de diseño y su mejoramiento.

La investigación hace parte del proyecto de investigación titulado "OPTIMIZACIÓN DEL PROCESO DE DISEÑO PARA PROYECTOS DE ARQUITECTURA SOSTENIBLE MEDIANTE DISEÑO INTEGRADO $Y$ BUILDING INFORMATION MODELING (BIM), BAJO UN AMBIENTE DE COLABORACIÓN EXTREMA". Liderado por la Universidad La Gran Colombia Sede Bogotá en colaboración con la Universidad Colegio Mayor de Cundinamarca, Universidad del Bío Bío (Chile), Universidad de la República del Uruguay y distintas empresas del sector productivo.

\section{REFERENCIAS}

Ambiente, M., \& Madrid, C. (2010). Aportaciones para el diseño de una política energética española sostenible. Retrieved from http://istas.net/descargas/Doc Energia Completo v11_VERSION WEB.pdf
Asaee, S. R., Ugursal, V. I., \& Beausoleil-Morrison, I. (2019). Development and analysis of strategies to facilitate the conversion of Canadian houses into net zero energy buildings. Energy Policy, 126, 118-130. https://doi.org/10.1016/j.enpol.2018.10.055

Bustamante, W., Encinas, F., Martínez, P., Brahm, M., \& Ibaceta, I. (2009). Guia de diseño para la eficiencia energetica en la vivienda social. Gobierno de Chile, 203.

CITECTUBB, D. (2015). TDRe 10 Términos de referencia estandarizados con parámetros de eficiencia energética.

Collado, N., Himpe, E., González, D., \& Rueda, L. (2019). Retos para una definición de "Edificios de consumo energético casi nulo " Challenges for a definition of Nearly Zero Energy Buildings. 34, 321-329.

Forcael, E., Martínez-Rocamora, A., Sepúlveda-Morales, J., García-Alvarado, R., Nope-Bernal, A., \& Leighton, F. (2020). Behavior and Performance of BIM Users in a Collaborative Work Environment. Applied Sciences, $\quad$ 10(6), 2199 . https://doi.org/10.3390/app10062199

Gong, Y., Liu, F., Tian, Z., Wu, G., Ren, H., \& Guan, C. (2019). Evaluation of Mechanical Properties of Cross-Laminated Timber with Different Lay-ups Using Japanese Larch. Journal of Renewable Materials, $\quad 7(10), \quad 941-956$. https://doi.org/10.32604/jrm.2019.07354

Joanna, A., Sartori, I., Napolitano, A., Marszal, A. J., Pless, S., \& Torcellini, P. (2010). Aalborg Universitet Criteria for Definition of Net Zero Energy Buildings.

Kwok, J., Wong, W., \& Zhou, J. (2015). Automation in Construction Enhancing environmental sustainability over building life cycles through green BIM: A review. Automation in Construction, 57, 156-165. https://doi.org/10.1016/j.autcon.2015.06.003

Kurnitski, J., Saari, A., Kalamees, T., Vuolle, M., Niemelä, J., \& Tark, T. (2011). Cost optimal and nearly zero (nZEB) energy performance calculations for residential buildings with REHVA definition for $n Z E B$ national implementation. Energy and Buildings, 43(11), 3279-3288. https://doi.org/10.1016/j.enbuild.2011.08.033

Laguarda Mallo, M. F., \& Espinoza, O. A. (2014). Outlook for Cross-Laminated Timber in the United States. BioResources, $9(4), \quad 7427-7443$. https://doi.org/10.15376/biores.9.4.7427-7443

Marszal, A. J., Heiselberg, P., Bourrelle, J. S., Musall, E., Voss, K., Sartori, I., \& Napolitano, A. (2011). Zero Energy Building - A review of definitions and calculation methodologies. Energy and Buildings, 43(4),

https://doi.org/10.1016/j.enbuild.2010.12.022

Minvu, M. de V. y U. de C. (2018). Estándares de Construcción Sustentable para Viviendas de Chile (M. División Técnica de Estudio y Fomento Habitacional - Ditec (ed.)).

Parasonis, J., Keizikas, A., Endriukaitytè, A., \& Kalibatienè, D. (2012). Architectural Solutions to Increase the Energy Efficiency of Buildings. Journal of Civil Engineering and Management, 18(1), 71-80. https://doi.org/10.3846/13923730.2011.652983 
Pei, S., van de Lindt, J. W., Popovski, M., Berman, J. W., Dolan, J. D., Ricles, J., Sause, R., Blomgren, H., \& Rammer, D. R. (2016). Cross-Laminated Timber for Seismic Regions: Progress and Challenges for Research and Implementation. Journal of Structural Engineering,

142(4). https://doi.org/10.1061/(ASCE)ST.1943$541 X .0001192$

Piderit, M. B., Vivanco, F., van Moeseke, G., \& Attia, S. (2019). Net zero buildings-A framework for an integrated policy in Chile. Sustainability (Switzerland), 11(5), 1-18. https://doi.org/10.3390/su11051494

Trebilcock, M. (2009). Proceso de Diseño Integrado: nuevos paradigmas en arquitectura sustentable. Arquitetura Revista, 5(2), 65-75. https://doi.org/10.4013/arq.2009.52.01 